www.mdpi.com/journal/sensors

Conference Proceedings Paper-Sensors and Applications

\title{
Assessment of Polysilicon film Properties through On-Chip Tests
}

\section{Ramin Mirzazadeh *, Aldo Ghisi and Stefano Mariani}

Politecnico di Milano, Department of Civil and Environmental Engineering Piazza Leonardo da Vinci, 32, 20133 Milano (Italy); E-Mails: aldo.ghisi@polimi.it (A.G.); stefano.mariani@polimi.it (S.M.)

* Author to whom correspondence should be addressed; E-Mail: ramin.mirzazadeh@polimi.it; Tel.: +39-022399-4310; Fax: +39-022399-4300.

Published: 5 November 2015

\begin{abstract}
Due to the increasing demand of miniaturization of MEMS devices, the characteristic size (e.g. the width) of some mechanical components may become comparable to that of a silicon grain. Therefore, the relevant effective mechanical properties can vary significantly from one device to another. In this work, through on-chip tests we investigate the behavior of polysilicon films using standard electrostatic actuation/sensing. The outcomes of the experimental campaign are then compared to those obtained with an analytical reduced-order model of the moving structure, and to coupled electro-mechanical simulations accounting for the polycrystalline morphology of the silicon film. These two models are adopted to bilaterally bound the experimental data up to pull-in, and to assess the scattering induced by the random orientation of the crystal lattice of each grain in slender parts of the devices.
\end{abstract}

Keywords: polysilicon film; morphology uncertainties; coupled electro-mechanical simulations; reduced order model

\section{Introduction}

Polysilicon films are widely used in micro-electro-mechanical-systems (MEMS) for the moving, structural parts of the devices. However, due to increasing miniaturization of MEMS technologies, some of the mechanical components can feature a characteristic size comparable to that of the polysilicon film itself (i.e. the silicon grain size). As silicon is an anisotropic material, crystal 
orientation can play a significant role in defining the elastic behavior [1] of each single device; overall, sensitivity to imperfections more and more becomes a crucial aspect to be investigated.

To assess the relevance of the silicon grain morphology, an on-chip test is proposed to investigate the mechanical response of a slender beam. In this paper, we compare experimental data with results of a reduced-order analytical model and of coupled electro-mechanical numerical simulations.

\section{Experimental}

On-chip tests have been carried by exploiting the structural configuration reported in Figure 1. A massive plate, denoted as rotor in the picture, is linked to the anchor point through a single beam, which behaves like a cantilever and therefore results to be free from relevant residual stresses. The system can be actuated either through the lateral stator, so as to mainly induce a translation of the rotor, or through the two anti-symmetric stators located along the top and bottom sides, so as to induce an in-plane rotation of the rotor. Because of the design geometry, translation of the plate leads to a shear deformation of the beam (somehow accompanied by bending deformation), whereas rotation leads to a pure bending of the beam itself. Sensing is obtained thanks to the same stators, measuring the capacitance change during the motion of the rotor induced by the applied voltage.

Geometrical parameters of the set up are summarized in Table 1. It can be seen that the geometry is set so as only the beam length is varied from one set up to another. This has been devised to capture micromechanical effects induced by: polysilicon morphology; shear or bending loading, and resultant deformation mode; the length of beam regions affected by the stress intensification caused by the reentrant corners at the connections with the anchor and plate; overetch across the beam thickness.

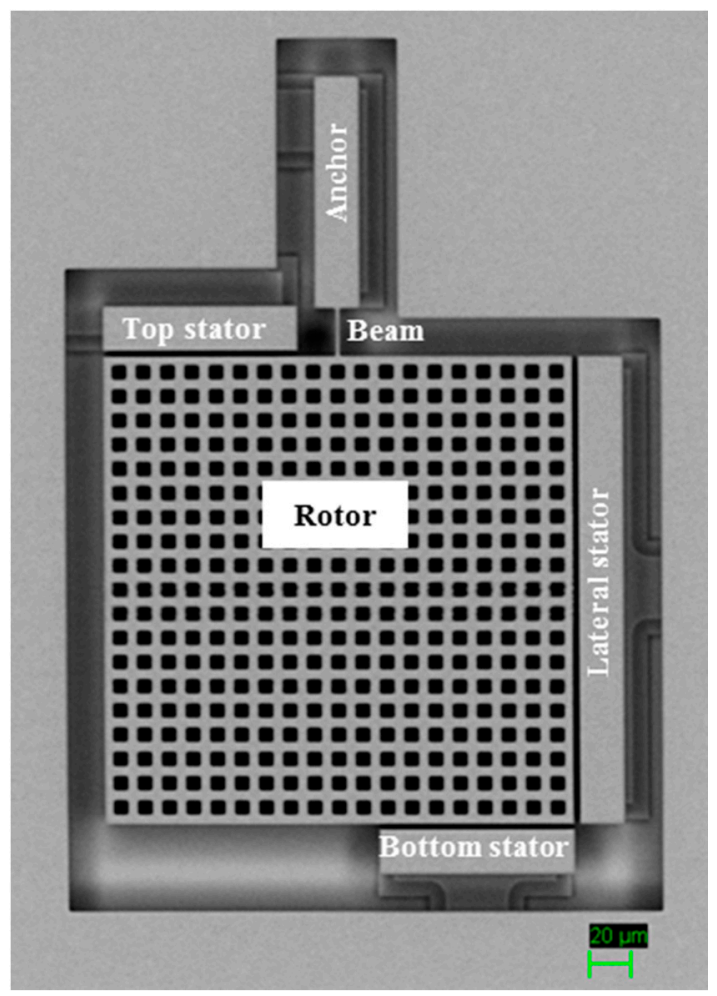

Figure 1. SEM image of the device for on-chip tests. 
In what follows, we focus only on static tests, i.e. on a motion of the plate induced by a smooth and slow variation in time of the applied voltage, and do not look at prominent inertial effects on the moving parts. Some exemplary results are reported in Figure 2 for the devices featuring a beam length of $20 \mu \mathrm{m}$; here, results are reported in terms of measured capacitance change (in $\mathrm{pF}$ ) vs the applied voltage at the top and bottom stators. Actuation has been provided to the system up to pull-in in two cases, measuring the related voltage as $39.25 \mathrm{~V}$ and $39.75 \mathrm{~V}$ : in these cases, the electrical short circuit leads to damage of the devices, and no further tests were possible for them. In all the other cases, to assess the repeatability of the curves several tests have been conducted on each device by increasing the voltage and then decreasing it back to the initial, undeformed configuration.

An excellent repeatability can be observed, as subsequent loading and unloading paths cannot be distinguished at the scale of the graph. On the other hand, the response of the different devices looks rather scattered. Due to the peculiar geometry of the moving parts, this is induced by small differences in the beam configuration either in terms of width $h$ and length $l$, or in the grain arrangement. At this scale, in fact, also geometric features have to be dealt with as stochastic variables, due to the possible effects of overetch. As for the film morphology, the Thelma process [2] adopted to get the moving structure leads to an average grain size of about $500 \mathrm{~nm}$, with the axis of epitaxial growth (almost) perpendicular to the substrate surface, i.e. perpendicular to the plane reported in Figure 1.

Table 1. Geometric dimensions of the device.

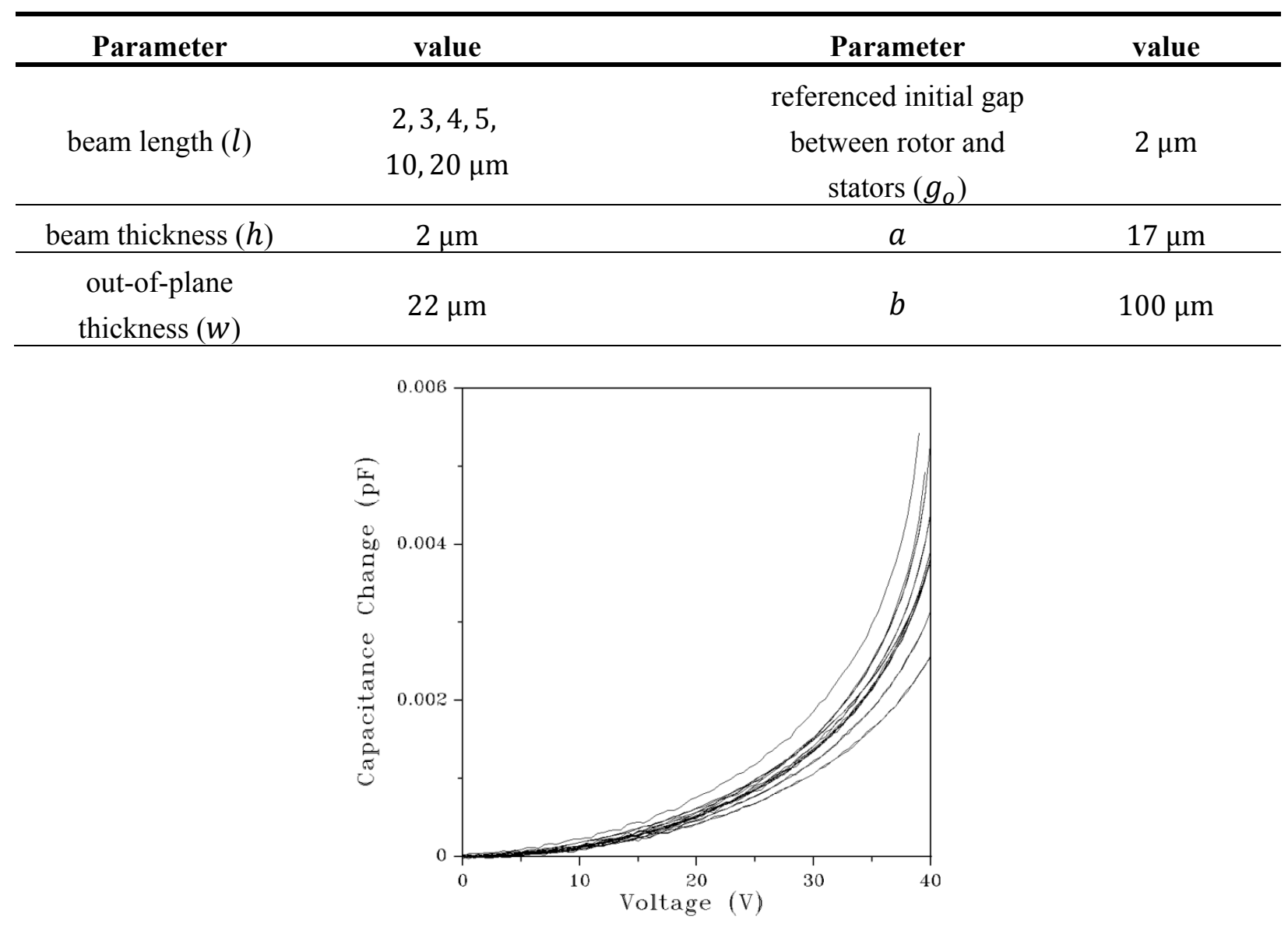

Figure 2. Measured device response in terms of capacitance change versus applied voltage $(l=20 \mu \mathrm{m})$. 


\section{Test Modeling}

The scattering of the experimental data has been assessed through an analytical beam bending reduced-order model of the structure, and through coupled electro-mechanical numerical simulations to also account for possible fringe field effects in the electrical medium (the air surrounding the structure).

\subsection{Reduced-Order Model}

The suspension beam is modeled according to the Euler-Bernoulli theory, i.e. shear deformations are neglected all over its length. The proof mass is instead considered as a rigid plate, undergoing only rotation and lateral displacement provided by beam bending. Electrical fringe fields near the conductors are neglected. The capacitance $C$ along the parallel plates in the current configuration is computed accounting to:

$$
C=\int \frac{\varepsilon w}{g} d s
$$

where $\varepsilon$ is the air permittivity, $w$ is the out-of-plane thickness of the beam. $g$ is the local gap between stators and rotor, which can be a function of the position (in the equation, of the coordinate $s$ along the plate surface). Accordingly, in case of bending actuation through the top and bottom stators the capacitance values as function of the rotation of the plate, $\theta$ are given by:

$C_{\text {top }}=\int_{a+\frac{\theta l}{2}}^{b+\frac{\theta l}{2}} \frac{\varepsilon w}{g+x \sin (\theta)} d x, \quad C_{\text {bottom }}=\int_{a-\frac{\theta l}{2}-2 b \theta}^{b} \frac{\varepsilon w}{g+2 b(1-\cos (\theta))+y \sin (\theta)} d y$

where $a$ and $b$ are provided in Figure 3, and $x$ and $y$ play now the role of the local coordinate $s$ along the actuated surfaces. The electrostatic bending moment for a given capacitance can be obtained by differentiation of the electrostatic energy with respect to the rotation angle $\theta$, namely:

$$
M_{\text {elec }}=\frac{1}{2} V^{2} \frac{d C}{d \theta}
$$

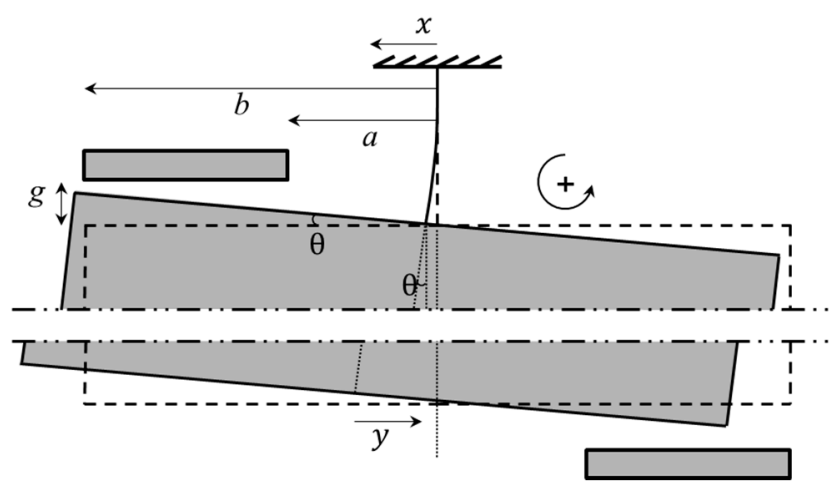

Figure 3. Sketch of the deformed configuration of the device, during bending actuation through top and bottom stators.

The total electrostatic moment induced by the top and bottom capacitors is equilibrated by the mechanical term provided by the (elastic) energy stored in the beam while bending; the value of the 
angle $\theta$ at equilibrium for any given voltage can then be calculated. Finally, the capacitance change between the deformed and initial configurations of the device can be calculated with Eq. (2) as a function of the applied voltage.

\subsection{Numerical Simulation}

Coupled electrostatic-mechanical analyses have been carried through a commercial code (ANSYSAPDL [4]). As already mentioned, scattering of the device response can be induced by the polycrystalline morphology of the silicon film. This effect was looked for an purpose and the (inplane) width of the suspension spring was set to be a few times the characteristic size of a single silicon grain. The effect of the mismatch of lattice orientations in adjacent grains is enhanced, leading to local stress intensification close to grain boundaries and to possible locking (i.e. stiffening) during motion.

The random morphology has been obtained through a plane Voronoi tessellation featuring an average grain size of about $500 \mathrm{~nm}$, whose shape perfectly matches the beam one plus two regions at the anchor and the proof mass sides, where some sort of stress diffusion is expected to take place due to the reentrant corners, see Figure 4. Randomness of the film morphology is assured by tiling a large region of the plane, and then extracting from it the morphology with a random placement. Each grain is modelled as an elastic orthotropic body, with an in-plane crystal orientation assigned randomly. For comparison purposes, the beam film has been also modelled as in-plane isotropic, like the other moving parts of the device, with the following overall elastic properties: Young's modulus $E=$ 149.3 GPa and Poisson's ratio $v=0.172$, see [3].

The simulations have been carried out in two steps. To account for the finite changes of the geometry of the electrical domains between capacitors, the displacement field relevant to a given applied voltage at capacitors is first obtained; then the geometry is updated and an electrostatic step is performed to obtain the relevant value of the capacitance between conductors.

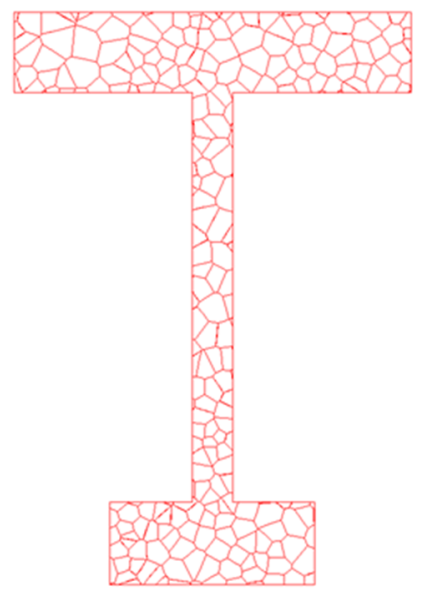

Figure 4. An exemplary polysilicon morphology for the beam region.

To bound the effect of film morphology, two analyses are first performed for each test set up. In these simulations, the polysilicon morphology is neglected and the beam is modeled as homogeneous and orthotropic, with the longitudinal axis aligned with either crystal orientation $<100>$ or $<110>$, 
respectively called in this work the "compliant" and "stiff" ones. Additionally, as mentioned a reference solution has been also computed by assuming the polysilicon to be in-plane isotropic; such one is supposed to be of interest only for extremely thick beam, when enough grains are included along its width and asymptotic homogenized mechanical properties prove sufficient.

Next, to properly account for the spreading of data due to fine microstructural features, a Monte Carlo simulation has been run, with 100 different crystal morphologies. The results of these two series of simulations are shown together with the measurements in Figure 5.
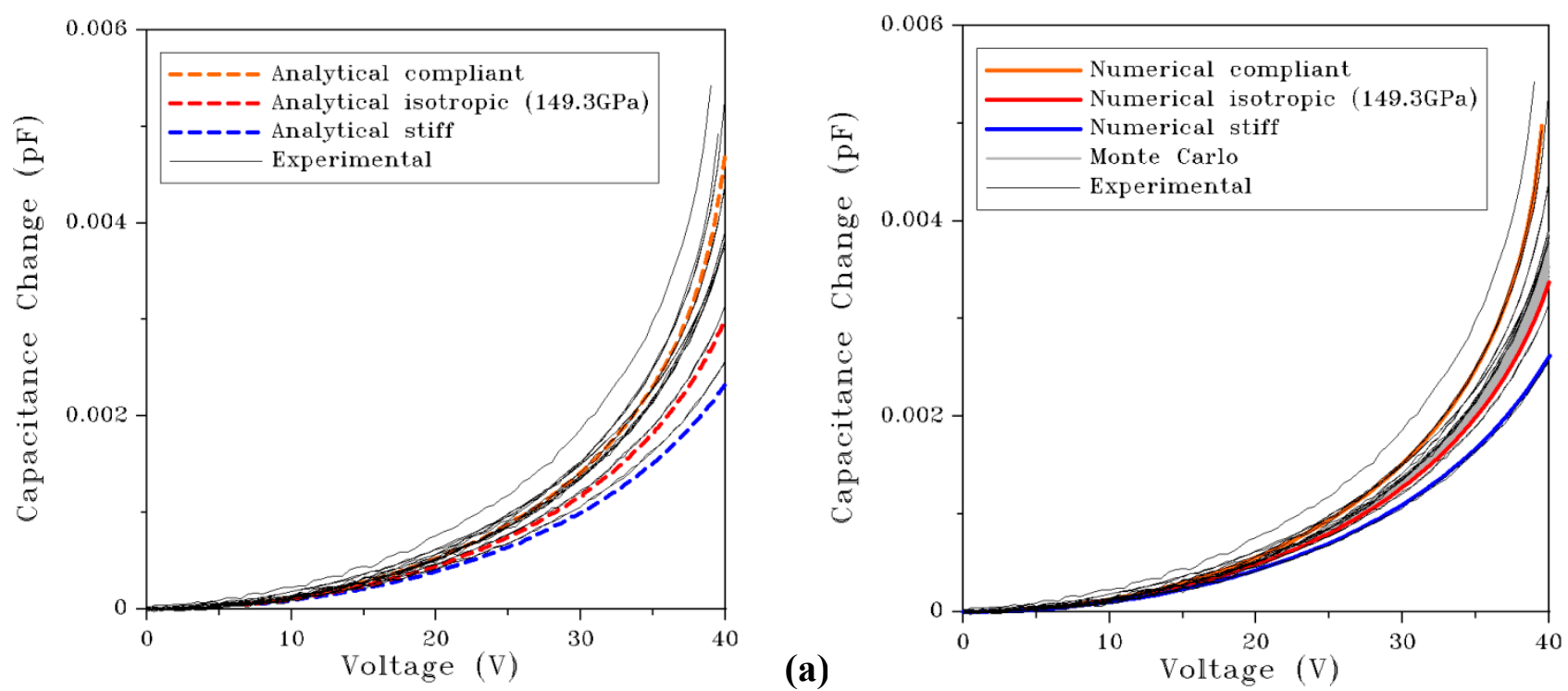

(b)

Figure 5. Comparison between measurement and outcomes (a) of the analytical model and (b) of the numerical simulations.

\subsection{Discussion}

The analytical model (see Figure 5a) can predict reasonably well the device behavior. However, since the adopted kinematics of the beam is in accordance with the classical beam theory, the model overestimates the stiffness and therefore underestimates the capacitance change; overall, all the plots are somehow moved to the right of the experimental ones in the graphs. The numerical model (see Figure 5b) obviously provides more accurate bounds on the measurements, as also shear deformations in the beam are allowed for.

Independently of the method used to provide an interpretation of the behavior of the device, it can be seen that all the measurements but one are between the bounds. The scattering of the numerical results associated to the random morphology of polysilicon provides a good estimation for the possible scattering of the real response of the devices. The out-of-bounds response needs to be finally considered. It has been already mentioned that polysilicon morphology is not the only source of uncertainties at this scale; further to that, overetch may play a prominent role if the beam is as (inplane) thin as the designed one. Since overetch is known to be not homogeneous all over a wafer, and to also depend on the geometry of the device (specifically, on the area to be laterally etched), further simulations and measurement look necessary to assess its impact on the spreading of data. 


\section{Conclusions/Outlook}

We carried out electrostatic on-chip tests on MEMS devices featuring an extremely thin polysilicon beam, whose in-plane width is on the order of the silicon grain. The effect of randomness of grain morphology in the beam on the capacitance change vs applied voltage response has been assessed by comparing the experimental data with the outcome of an analytical, simplified (or reduced-order) model and of coupled electro-mechanical finite element simulations. In the analytical model, the beam has been assumed to deform according to the Euler-Bernoulli theory; in the finite element simulations, account has been taken of grain morphology in the beam through Voronoi tessellations. Both the approaches have provided insights into the silicon grain effects, which were not negligible due to the designed test set up.

Results will be further extended in future research, by comparing the responses of devices featuring different slenderness $l / w$ ratios.

\section{Acknowledgments}

Financial support provided by STMicroelectronics through project MaRe (MAterail REliability) is gratefully acknowledged.

\section{Conflicts of Interest}

The authors declare no conflict of interest.

\section{References}

1. Hopcroft, M. A.; Nix, W. D.; Kenny, T. W. What is the Young's Modulus of silicon? Journal of Microelectromechanical Systems 2010, 19, 229-238.

2. Corigliano, A.; De Massi, B.; Frangi, A.; Comi, C.; Villa, A. ; Marchi, M. Mechanical Characterization of Polysilicon Through On-Chip Tensile Tests. Journal of Microelectromechanical Systems 2004, 13, 200-219.

3. Mariani, S.; Martini, R.; Ghisi, A.; Corigliano, A.; Beghi, M. Overall elastic properties of polysilicon films: a statistical investigation of the effects of polycrystal morphology. International Journal for Multiscale Computational Engineering 2011, 9, 327-346.

4. ANSYS reference guide, release 13.

(C) 2015 by the authors; licensee MDPI, Basel, Switzerland. This article is an open access article distributed under the terms and conditions of the Creative Commons Attribution license (http://creativecommons.org/licenses/by/4.0/). 